\title{
Sagittamides A and B. Polyacetoxy Long-Chain Acyl Amino acids from a Didemnid Ascidian
}

\author{
Sarah C. Lievens and Tadeusz F. Molinski* \\ Department of Chemistry, University of California, Davis, CA 95616 \\ tfmolinski@ucdavis.edu \\ Supplementary Information Contents \\ S2: Underwater Photo of 01-133 \\ S3: NMR Assignment of 1 \\ S4: $400 \mathrm{MHz}{ }^{1} \mathrm{H}$ NMR of $1\left(\mathrm{CD}_{3} \mathrm{OD}\right)$ \\ S5: $100 \mathrm{MHz}{ }^{13} \mathrm{C}$ NMR of $1\left(\mathrm{CD}_{3} \mathrm{OD}\right)$ \\ S6: $600 \mathrm{MHz}$ DFQ-COSY of $1\left(\mathrm{CD}_{3} \mathrm{OD}\right)$ \\ S7: $600 \mathrm{MHz} \mathrm{HSQC}$ of $1\left(\mathrm{CD}_{3} \mathrm{OD}\right)$ \\ S8: $600 \mathrm{MHz} \mathrm{HMBC}$ of $1\left(\mathrm{CD}_{3} \mathrm{OD}\right)$ \\ S9: $600 \mathrm{MHz}{ }^{1} \mathrm{H}$ NMR of 1 ( $d_{6}$-DMSO) \\ S10: $600 \mathrm{MHz}$ DFQ-COSY of $1\left(d_{6}\right.$-DMSO) \\ S11: $600 \mathrm{MHz}$ HMQC of 1 ( $d_{6}$-DMSO) \\ S12: $600 \mathrm{MHz}$ HMBC of 1 ( $d_{6}$-DMSO) \\ S13: $400 \mathrm{MHz}{ }^{1} \mathrm{H}$ NMR of 3 \\ S14: NMR Assignment of 2 \\ S15: $400 \mathrm{MHz}{ }^{1} \mathrm{H}$ NMR of 2 \\ S16: $400 \mathrm{MHz}$ COSY of 2 \\ S17: $400 \mathrm{MHz}$ HSQC of 2 \\ S18: $600 \mathrm{MHz}$ Long-Range COSY of 2 \\ S19: 600 MHz HSQC-TOCSY of 2
}


Supplementry Table 1: NMR assignments of 1

\begin{tabular}{|c|c|c|c|c|c|c|}
\hline$\#$ & $\delta_{H}{ }^{a}$ & $\delta_{C}^{b}$ & $\delta_{H}^{c}$ & $\delta_{C}^{d}$ & COSY & НМВС \\
\hline 1 & & 175.9 & & 171.9 & & \\
\hline 2 & $2.27(2 \mathrm{H}, \mathrm{m})$ & 36.3 & $2.13(2 \mathrm{H}, \mathrm{t}, 6.9)$ & 34.5 & 3 & $1,3,4$ \\
\hline $3 a$ & $1.52(1 \mathrm{H}, \mathrm{m})$ & 23.0 & $1.35(2 \mathrm{H}, \mathrm{m})$ & 21.2 & & $1,4,5$ \\
\hline $3 b$ & $1.67(1 \mathrm{H}, \mathrm{m})$ & & & & & \\
\hline $4 a$ & $1.59(1 \mathrm{H}, \mathrm{m})$ & 29.1 & $1.47(1 \mathrm{H}, \mathrm{m})$ & 28.6 & & $1,2,5$ \\
\hline $4 b$ & $1.63(1 \mathrm{H}, \mathrm{m})$ & & $1.57(1 \mathrm{H}, \mathrm{m})$ & & & \\
\hline 5 & $4.92(1 \mathrm{H}, \mathrm{m})$ & 73.4 & $4.87(1 \mathrm{H}, \mathrm{m})$ & 71.1 & 4,6 & $3,4,6$ \\
\hline 6 & $5.11(1 \mathrm{H}, \mathrm{dd}, 8.3,4.0)$ & 71.4 & $5.00(1 \mathrm{H}, \mathrm{m})$ & 69.8 & & $4,5,7,29$ \\
\hline 7 & $5.15(1 \mathrm{H}, \mathrm{dd}, 8.3,1.5)$ & 69.2 & $5.05(1 \mathrm{H}, \mathrm{m})$ & 67.1 & 6,8 & $5,6,31$ \\
\hline 8 & $5.42(1 \mathrm{H}, \mathrm{dd}, 9.9,1.5)$ & 68.4 & $5.33(1 \mathrm{H}, \mathrm{d}, 9.6)$ & 66.3 & 7,9 & $6,7,9,33$ \\
\hline 9 & $5.13(1 \mathrm{H}, \mathrm{dd}, 9.9,1.8)$ & 70.4 & $5.03(1 \mathrm{H}, \mathrm{m})$ & 68.6 & 8,10 & $8,10,35$ \\
\hline 10 & $4.82(1 \mathrm{H}, \mathrm{dt}, 6.8,1.8)$ & 71.9 & $4.80(1 \mathrm{H}, \mathrm{t}, 6.6)$ & 69.6 & 9,11 & $8,9,11,12,37$ \\
\hline 11 & $1.42(2 \mathrm{H}, \mathrm{m})$ & 31.8 & $1.35(2 \mathrm{H}, \mathrm{m})$ & 30.2 & & $10,12,13$ \\
\hline 12 & $1.32(2 \mathrm{H}, \mathrm{m})$ & 26.3 & $1.23(2 \mathrm{H}, \mathrm{br} \mathrm{s})$ & 24.5 & & 10,13 \\
\hline $13-23$ & $1.28(22 \mathrm{H}$, br s $)$ & 30.5 & $1.23(22 \mathrm{H}$, br s $)$ & 28.9 & $12-24$ & $13-23$ \\
\hline $24 \mathrm{a}$ & $1.59(1 \mathrm{H}, \mathrm{m})$ & 27.1 & $1.47(2 \mathrm{H}, \mathrm{m})$ & 25.2 & 23,25 & $23,25,26$ \\
\hline $24 b$ & $1.63(1 \mathrm{H}, \mathrm{m})$ & & & & & \\
\hline 25 & $2.24(2 \mathrm{H}, \mathrm{m})$ & 37.0 & $2.10(2 \mathrm{H}, \mathrm{t}, 7.2)$ & 35.1 & 24 & $23,24,26$ \\
\hline 26 & & 176.6 & & 172.2 & & \\
\hline 27 & & 172.4 & & 169.9 & & \\
\hline 28 & 2.01 & 21.0 & $1.98^{\mathrm{g}}$ & $20.4^{\mathrm{h}}$ & & \\
\hline 29 & & 171.7 & & 169.5 & & \\
\hline 30 & 2.02 & $21.1^{\mathrm{e}}$ & $1.98^{\mathrm{g}}$ & $20.5^{\mathrm{h}}$ & & 6 \\
\hline 31 & & 172.0 & & 169.7 & & \\
\hline 32 & 2.07 & $20.9^{f}$ & $2.00^{\mathrm{g}}$ & $20.5^{\mathrm{h}}$ & & 7 \\
\hline 33 & & 171.5 & & 169.4 & & \\
\hline 34 & 2.01 & 21.0 & $2.00^{\mathrm{g}}$ & $20.5^{\mathrm{h}}$ & & 8 \\
\hline 35 & & 171.7 & & 169.1 & & \\
\hline 36 & 2.08 & $21.0^{\mathrm{f}}$ & $2.01^{\mathrm{g}}$ & $20.6^{\mathrm{h}}$ & & 9 \\
\hline 37 & & 172.6 & & 169.8 & & \\
\hline 38 & 2.03 & $21.2^{\mathrm{e}}$ & $2.06^{\mathrm{g}}$ & $20.7^{\mathrm{h}}$ & & \\
\hline $1^{\prime}$ & & 175.0 & & 173.2 & & \\
\hline $2^{\prime}$ & $4.31(1 \mathrm{H}, \mathrm{d}, 5.6)$ & 59.1 & $4.12(1 \mathrm{H}, \mathrm{dd}, 8.4,5.4)$ & 57.0 & $3{ }^{\prime}$ & $1,1^{\prime}, 3^{\prime}, 4^{\prime}, 5^{\prime}$ \\
\hline $3^{\prime}$ & $2.15(1 \mathrm{H}, \mathrm{m}, 6.8)$ & 31.8 & $2.03(1 \mathrm{H}, \mathrm{m})$ & 29.7 & $4^{\prime}, 5^{\prime}$ & $1^{\prime}, 2^{\prime}, 4^{\prime}, 5^{\prime}$ \\
\hline 4 ' & $0.96(3 \mathrm{H}, \mathrm{d}, 6.8)$ & 18.5 & $0.86(3 \mathrm{H}, \mathrm{d}, 6.6)$ & 18.0 & $3^{\prime}$ & $2^{\prime}, 3^{\prime}, 5^{\prime}$ \\
\hline 5 , & $0.97(3 \mathrm{H}, \mathrm{d}, 6.8)$ & 19.8 & $0.86(3 \mathrm{H}, \mathrm{d}, 6.6)$ & 19.1 & $3^{\prime}$ & $2^{\prime}, 3^{\prime}, 4^{\prime}$ \\
\hline $\mathrm{NH}$ & & & $7.91(1 \mathrm{H}, \mathrm{d}, 8.4)$ & & & \\
\hline 1, & & 174.9 & & 173.4 & & \\
\hline 2 ', & $4.42(2 \mathrm{H}, \mathrm{dd} 8.2,5.0)$ & 52.9 & $4.16(2 \mathrm{H}, \mathrm{m})$ & 51.3 & 3, & $28,1^{\prime \prime}, 3^{\prime \prime}, 4^{\prime \prime}$ \\
\hline $3 a^{\prime \prime}$ & $1.75(1 \mathrm{H}, \mathrm{m})$ & 29.9 & $1.57(1 \mathrm{H}, \mathrm{m})$ & 28.2 & $2{ }^{\prime \prime}, 3{ }^{\prime \prime}, 4{ }^{\prime \prime}$ & $1^{\prime \prime}, 2^{\prime \prime}, 4^{\prime \prime}, 5^{\prime \prime}$ \\
\hline $3 b^{\prime \prime}$ & $1.98(1 \mathrm{H}, \mathrm{m})$ & & $1.75(1 \mathrm{H}, \mathrm{m})$ & & & \\
\hline 4, & $1.72(2 \mathrm{H}, \mathrm{m})$ & 25.3 & $1.57(2 \mathrm{H}, \mathrm{m})$ & 23.8 & $3^{\prime}, 5^{\prime}$ & 1, \\
\hline 5, & $2.95(2 \mathrm{H}, \mathrm{t}, 7.2)$ & 40.4 & $2.77(2 \mathrm{H}, \mathrm{t}, 6.9)$ & 38.4 & 4, & $3 ', 4$,' \\
\hline $\mathrm{NH}$ & & & $8.00(1 \mathrm{H}, \mathrm{d}, 7.2)$ & & & \\
\hline
\end{tabular}

${ }^{\mathrm{a}}$ Measured at $600 \mathrm{MHz}$ in $\mathrm{CD}_{3} \mathrm{OD}$ referenced to $\mathrm{CHD}_{2} \mathrm{OD}(\delta=3.30)$. ${ }^{\mathrm{b}}$ Measured at $600 \mathrm{MHz}$ in $\mathrm{CD}_{3} \mathrm{OD}$ referenced to $\mathrm{CD}_{3} \mathrm{OD}(\delta=49.0) .{ }^{\mathrm{c}}$ Measured at $600 \mathrm{MHz}$ in $d_{6}$-DMSO referenced to $d_{5}$-DMSO $(\delta=2.50)$. ${ }^{\mathrm{d}}$ From HSQC and HMBC measured at $600 \mathrm{MHz}$ in $d_{6^{-}}$DMSO referenced to $d_{5^{-}}$DMSO $(\delta=2.50)$ and $d_{6^{-}}$ DMSO $(\delta=39.5){ }^{\text {e,f,g,h }}$ These signals are interchangeable. 


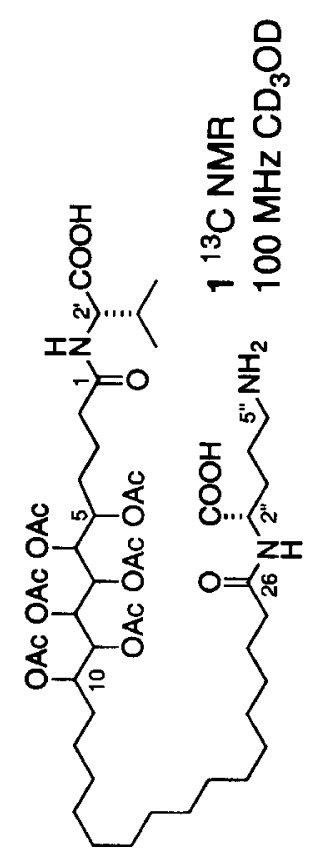

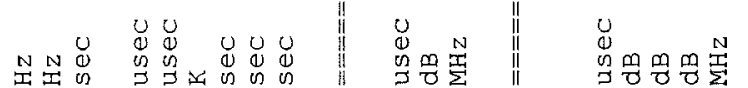
넌융요

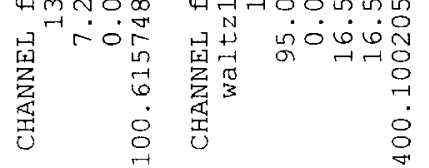
\|
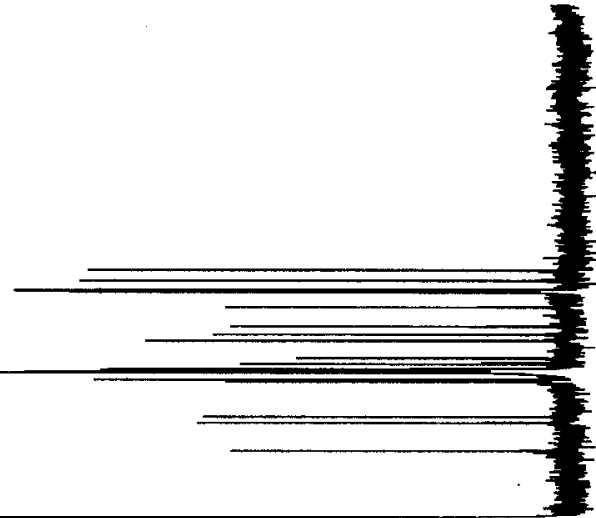

$-\stackrel{\square}{\square}$
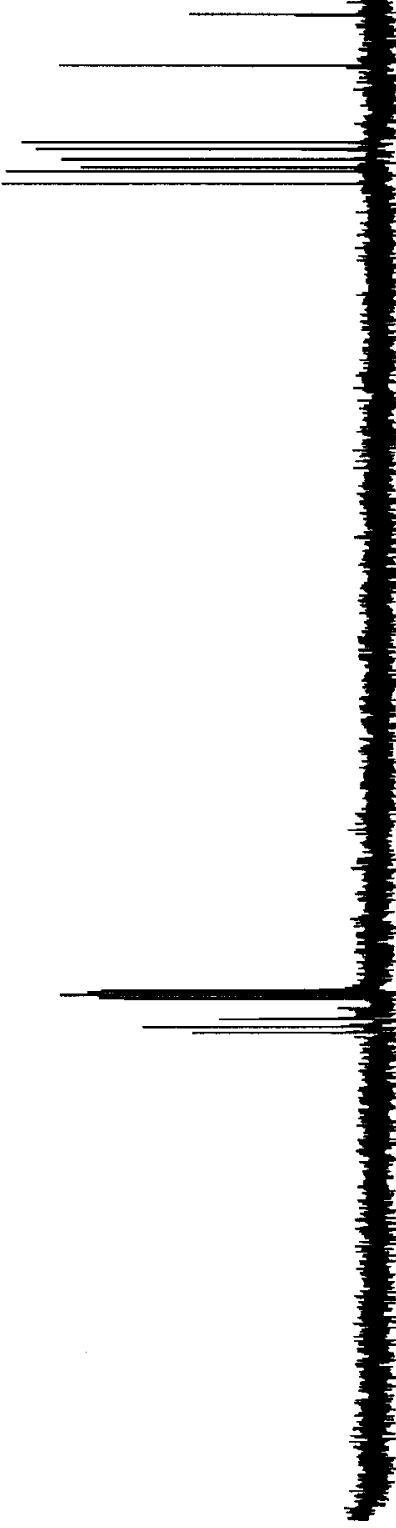

$-\infty$ 


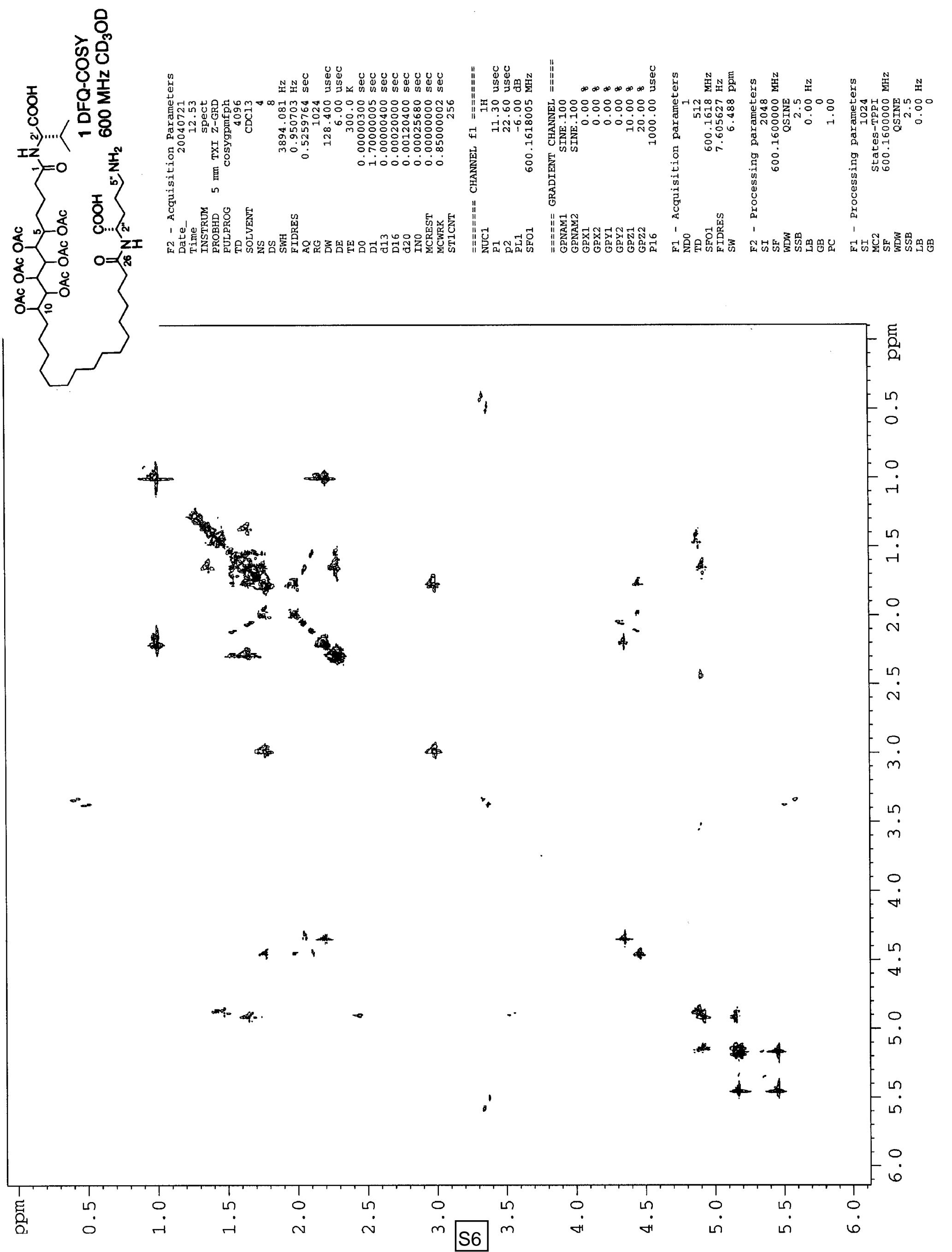



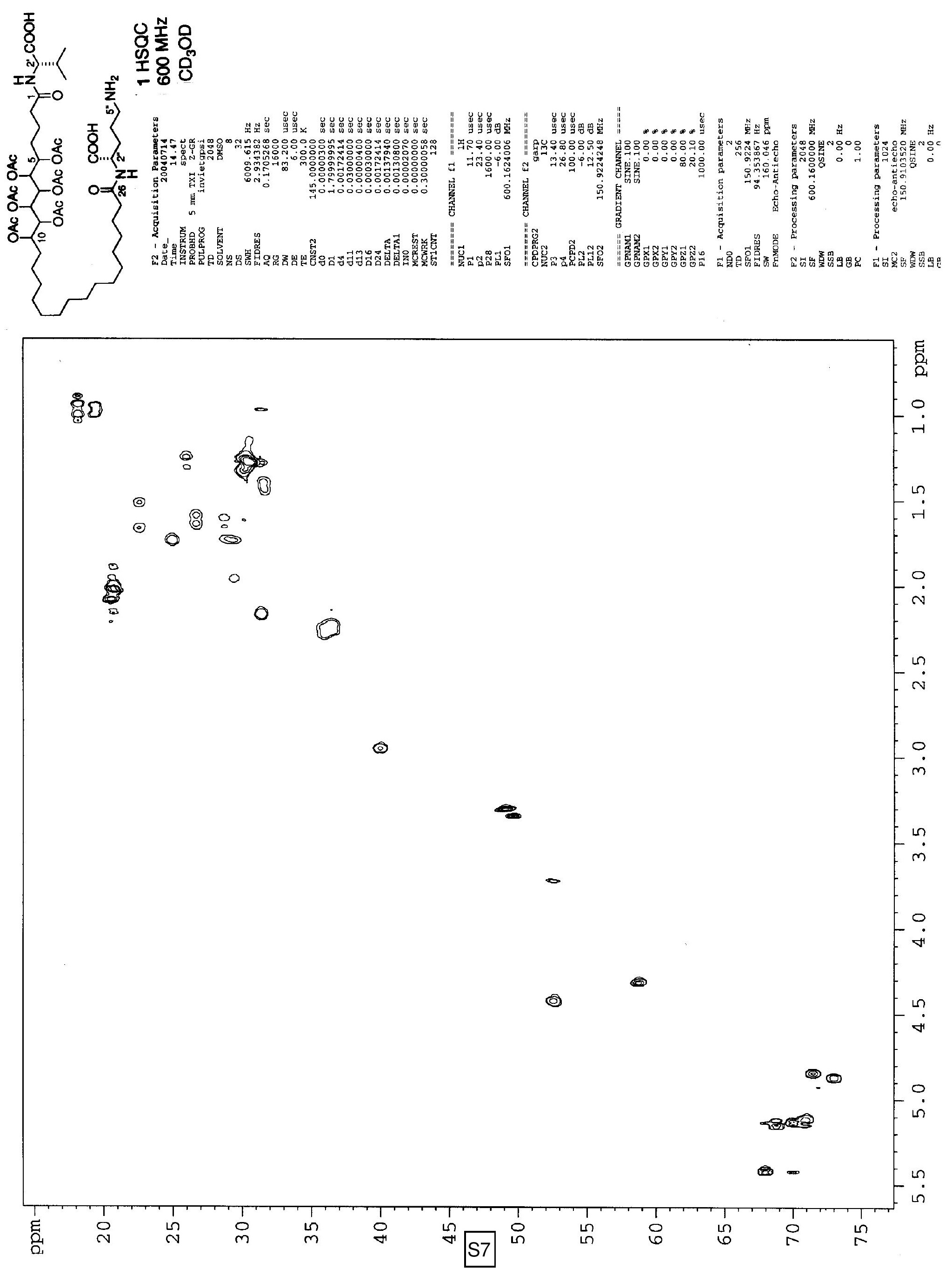

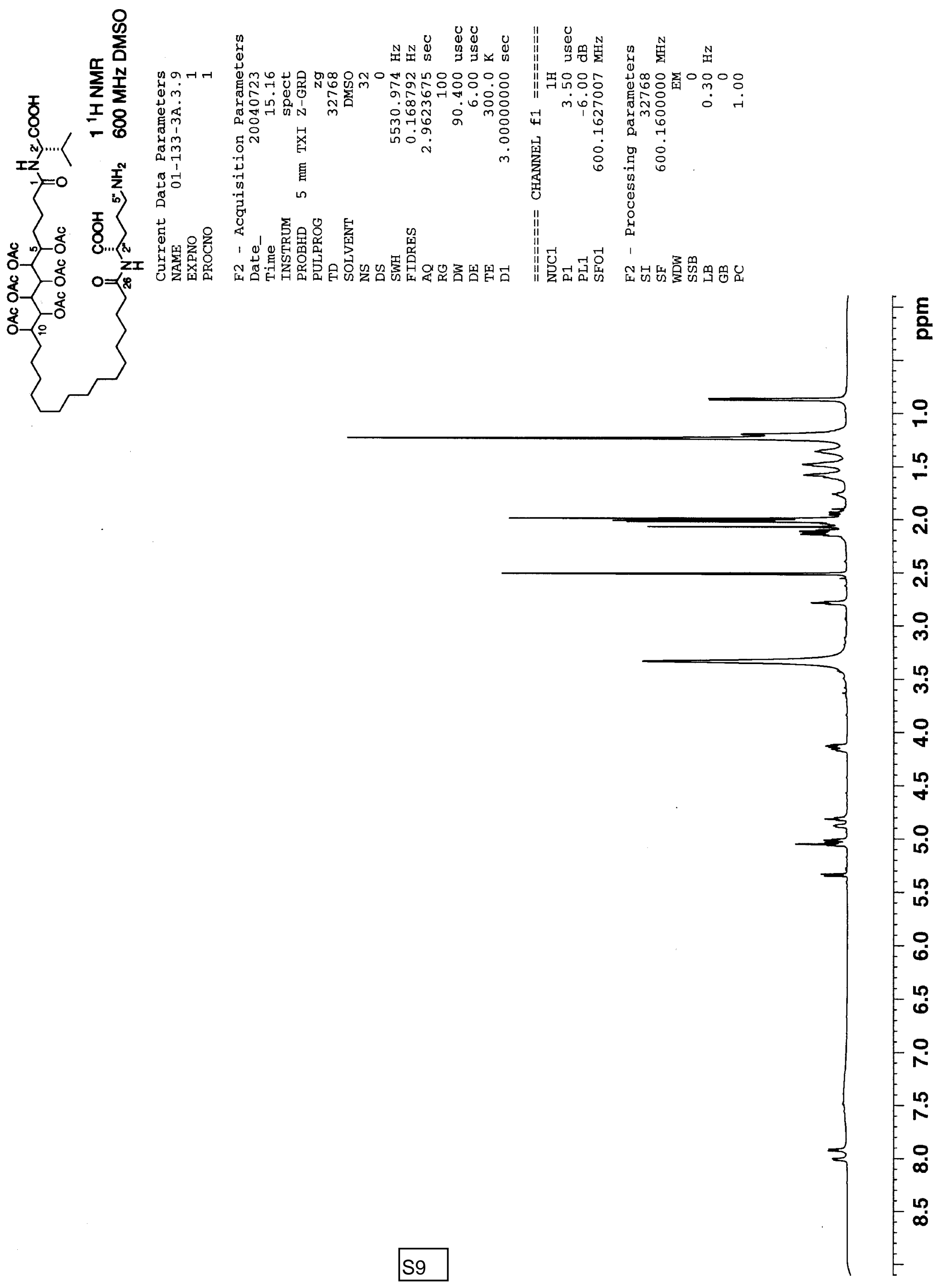


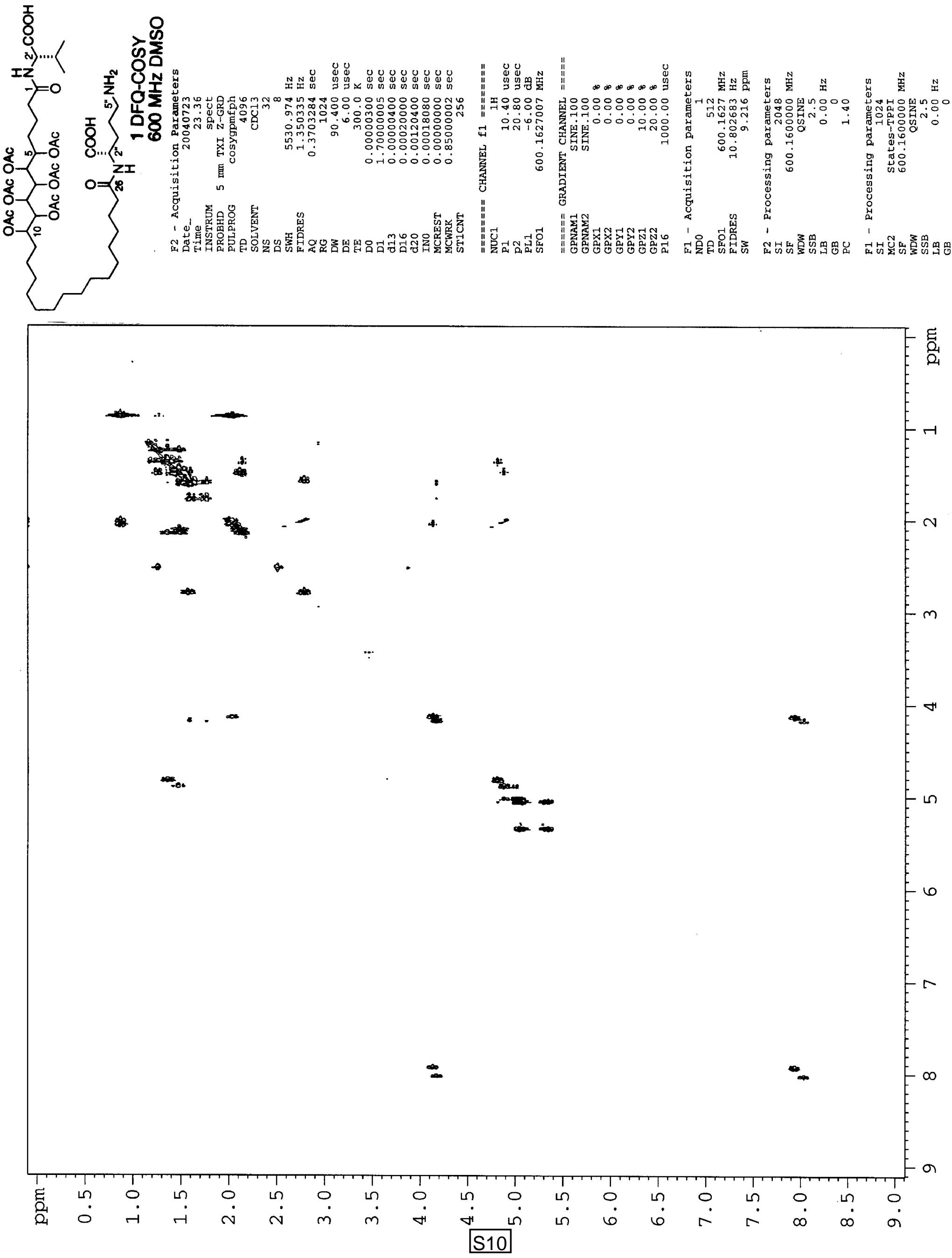




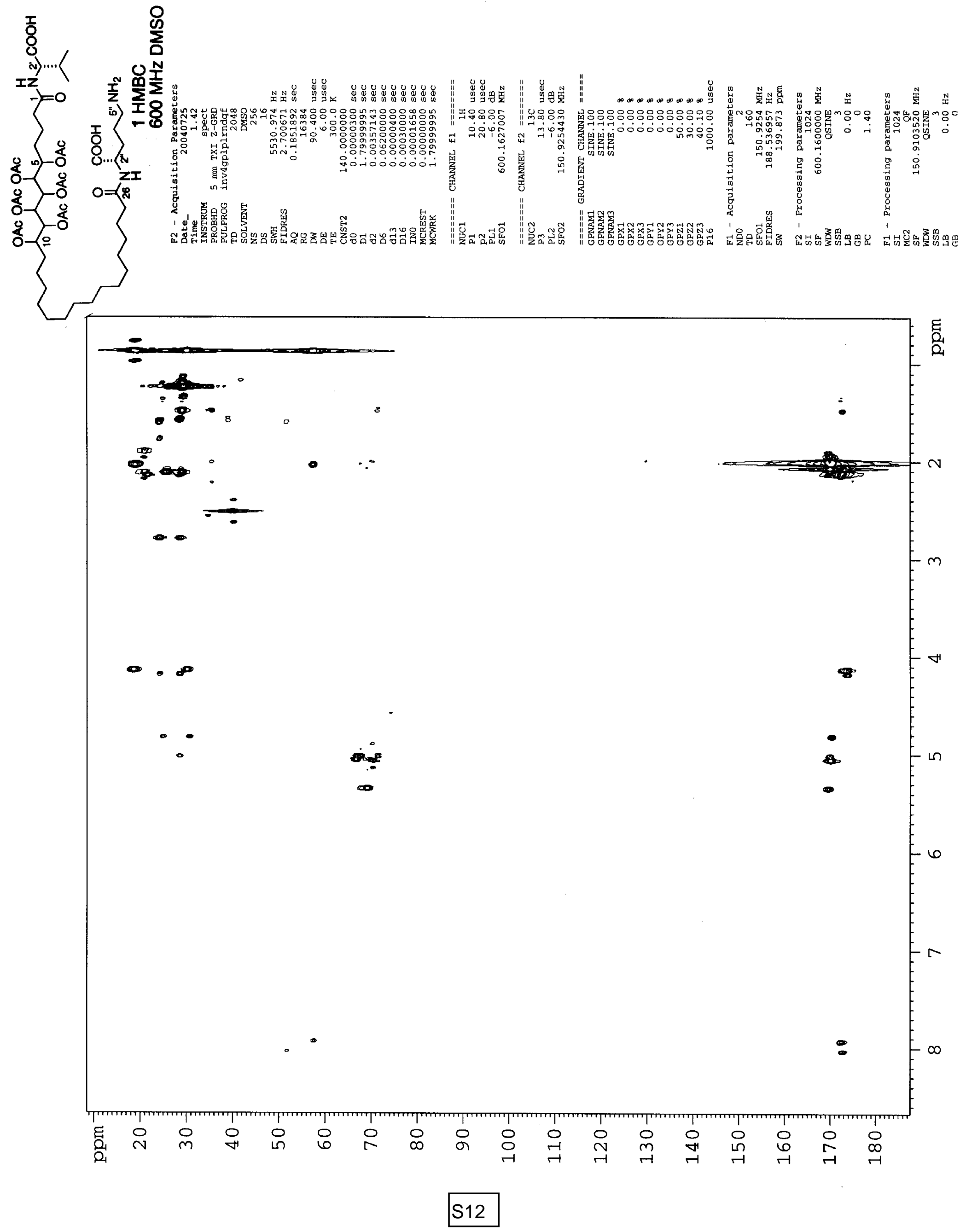




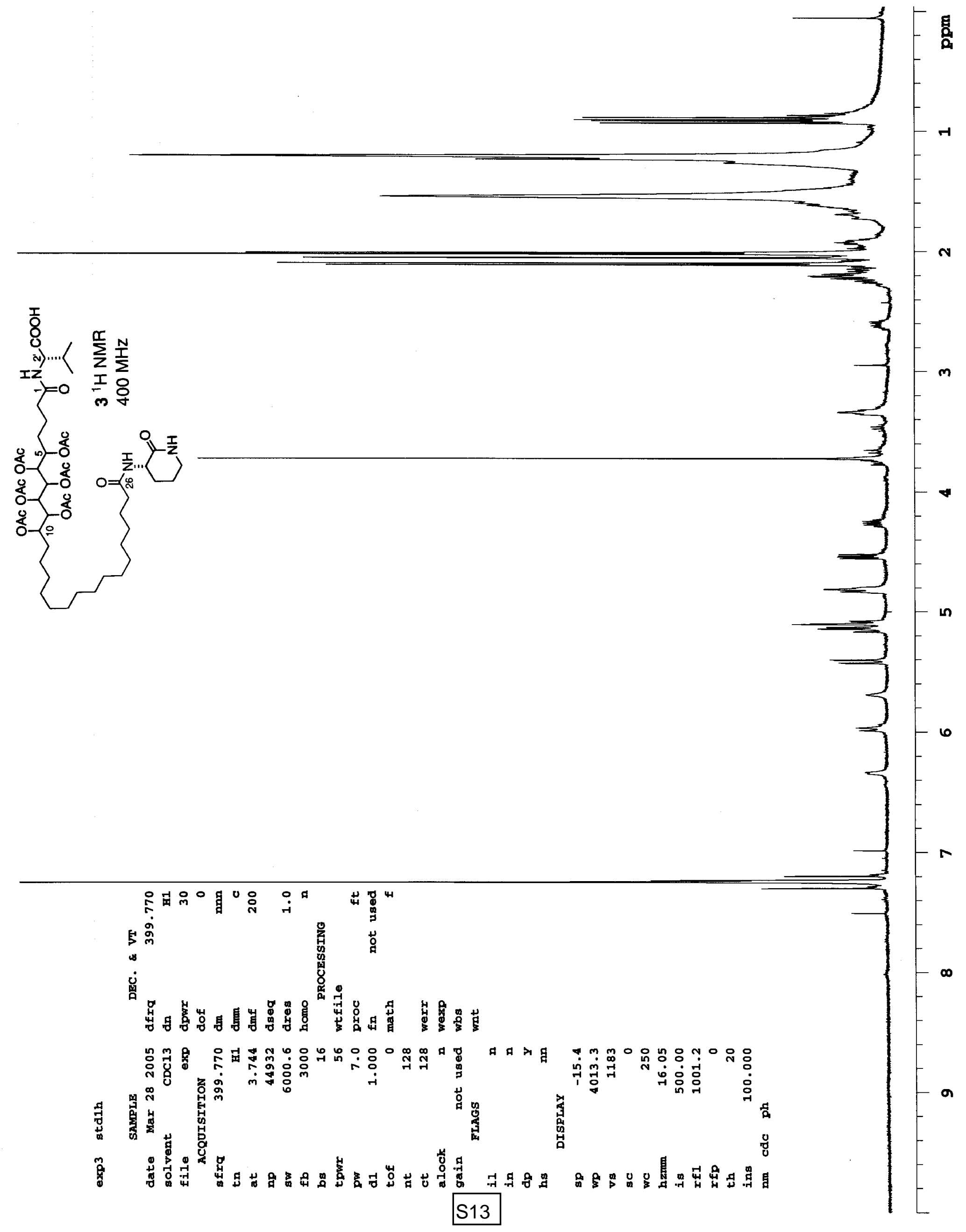


Supplementary Table 2: NMR Assignments for 2 in $\mathrm{CD}_{3} \mathrm{OD}$

\begin{tabular}{|c|c|c|c|c|}
\hline \# & $\delta_{H}{ }^{a}$ & $\delta_{C}{ }^{b}$ & $\operatorname{COSY}^{c}$ & HSQC-TOCSY ${ }^{\mathrm{d}}$ \\
\hline \multicolumn{5}{|l|}{1} \\
\hline 2 & $2.30(2 \mathrm{H}, \mathrm{m})$ & 36.0 & & 3,4 \\
\hline $3 a$ & $1.58(1 \mathrm{H}, \mathrm{m})$ & 22.7 & & 2 \\
\hline $3 b$ & $1.69(1 \mathrm{H}, \mathrm{m})$ & & & \\
\hline 4 & $1.73(2 \mathrm{H}, \mathrm{m})$ & 27.8 & & $2,3,5$ \\
\hline 5 & $5.22(1 \mathrm{H}, \mathrm{m})$ & 74.1 & 6 & $2,3,4$ \\
\hline 6 & $4.94(1 \mathrm{H}, \mathrm{dd}, 10.1,2.6)$ & 72.0 & 5,7 & \\
\hline 7 & $4.15(1 \mathrm{H}, \mathrm{dd}, 10.1,1.4)$ & 68.2 & 6,8 & 6 \\
\hline 8 & $4.99(1 \mathrm{H}, \mathrm{dd}, 9.7,1.4)$ & 71.2 & 7,9 & 9 \\
\hline 9 & $3.95(1 \mathrm{H}, \mathrm{dd}, 9.7,1.5)$ & 69.0 & 8,10 & 8 \\
\hline 10 & $4.76(1 \mathrm{H}, \mathrm{dt}, 6.8,1.5)$ & 73.2 & 9 & $11,12,13-23$ \\
\hline 11 & $1.42(2 \mathrm{H}, \mathrm{m})$ & 31.7 & & \\
\hline 12 & $1.32(2 \mathrm{H}, \mathrm{m})$ & 26.3 & & $11,13-23$ \\
\hline $13-23$ & $1.28(22 \mathrm{H}, \mathrm{br} \mathrm{s})$ & 30.4 & & $11,12,13-23,24,25$ \\
\hline 24 & $1.60(2 \mathrm{H}, \mathrm{m})$ & 26.8 & & $13-23,25$ \\
\hline 25 & $2.24(2 \mathrm{H}, \mathrm{m})$ & 36.8 & & $13-23,24$ \\
\hline \multicolumn{5}{|c|}{ (2) $(2 x, \ldots)$} \\
\hline \multicolumn{5}{|l|}{1 ' } \\
\hline 2' & $4.31(1 \mathrm{H}, \mathrm{d}, 6.0)$ & 59.0 & & $3^{\prime}, 4^{\prime}, 5^{\prime}$ \\
\hline $3^{\prime}$ & $2.17(1 \mathrm{H}, \mathrm{m}, 6.8)$ & 31.3 & & $2^{\prime}, 4^{\prime}, 5^{\prime}$ \\
\hline 4 ' & $0.97(3 \mathrm{H}, \mathrm{d}, 6.8)$ & 18.2 & & 2 \\
\hline 5 & $0.98(3 \mathrm{H}, \mathrm{d}, 6.8)$ & 19.2 & & 2 ' \\
\hline \multicolumn{5}{|c|}{ 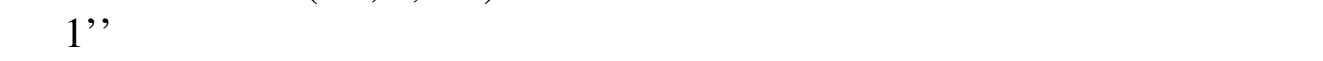 } \\
\hline $2 "$ & $4.43(2 \mathrm{H}, \mathrm{dd}, 8.0,5.2)$ & 53.2 & & $5 "$ \\
\hline $3 a^{\prime \prime}$ & $1.75(1 \mathrm{H}, \mathrm{m})$ & 29.9 & & $2^{\prime \prime}, 4^{\prime \prime}, 5^{\prime \prime}$ \\
\hline $3 b^{\prime \prime}$ & $1.98(1 \mathrm{H}, \mathrm{m})$ & & & \\
\hline $4 "$, & $1.75(2 \mathrm{H}, \mathrm{m})$ & 25.0 & & 2', 3', 5’' \\
\hline $5 "$ & $2.95(2 \mathrm{H}, \mathrm{t}, 7.0)$ & 39.9 & & $3 ", 4{ }^{\prime \prime}$ \\
\hline \multirow[t]{4}{*}{$\mathrm{OCOCH}_{3}$} & & 171.5 & & \\
\hline & & 171.7 & & \\
\hline & & 171.8 & & \\
\hline & & 172.0 & & \\
\hline \multirow[t]{4}{*}{$\mathrm{OCOCH}_{3}$} & $1.95(3 \mathrm{H}, \mathrm{s})$ & 20.5 & & \\
\hline & $1.97(3 \mathrm{H}, \mathrm{s})$ & 20.5 & & \\
\hline & $1.98(3 \mathrm{H}, \mathrm{s})$ & 20.5 & & \\
\hline & $1.99(3 \mathrm{H}, \mathrm{s})$ & 20.5 & & \\
\hline
\end{tabular}

\footnotetext{
${ }^{a}$ Measured at $400 \mathrm{MHz}$ in $\mathrm{CD}_{3} \mathrm{OD}$, referenced to $\mathrm{CHD}_{2} \mathrm{OD}(\delta=3.30) .{ }^{\mathrm{b}}$ Measured from HSQC and HMBC recorded at $400 \mathrm{MHz}$ in $\mathrm{CD}_{3} \mathrm{OD}$, referenced to $\mathrm{CD}_{3} \mathrm{OD}(\delta=49.0) .{ }^{c}$ Recorded in $\mathrm{CD}_{3} \mathrm{OD}$ from $400 \mathrm{MHz}$ $\mathrm{COSY}$ and $600 \mathrm{MHz}$ LR-COSY. ${ }^{\mathrm{d}}$ Recorded in $\mathrm{CD}_{3} \mathrm{OD}$ at $600 \mathrm{MHz}$.
} 

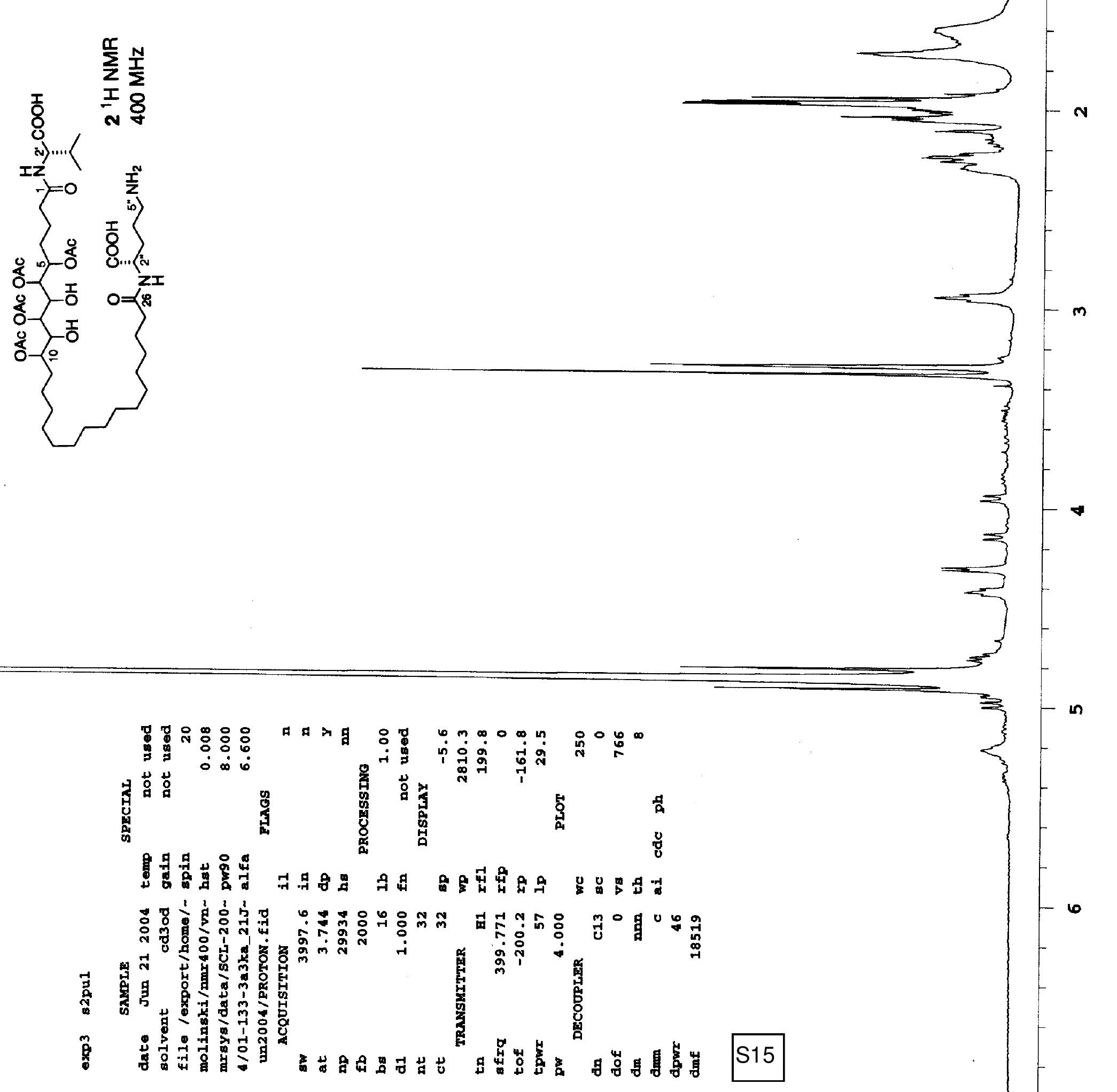


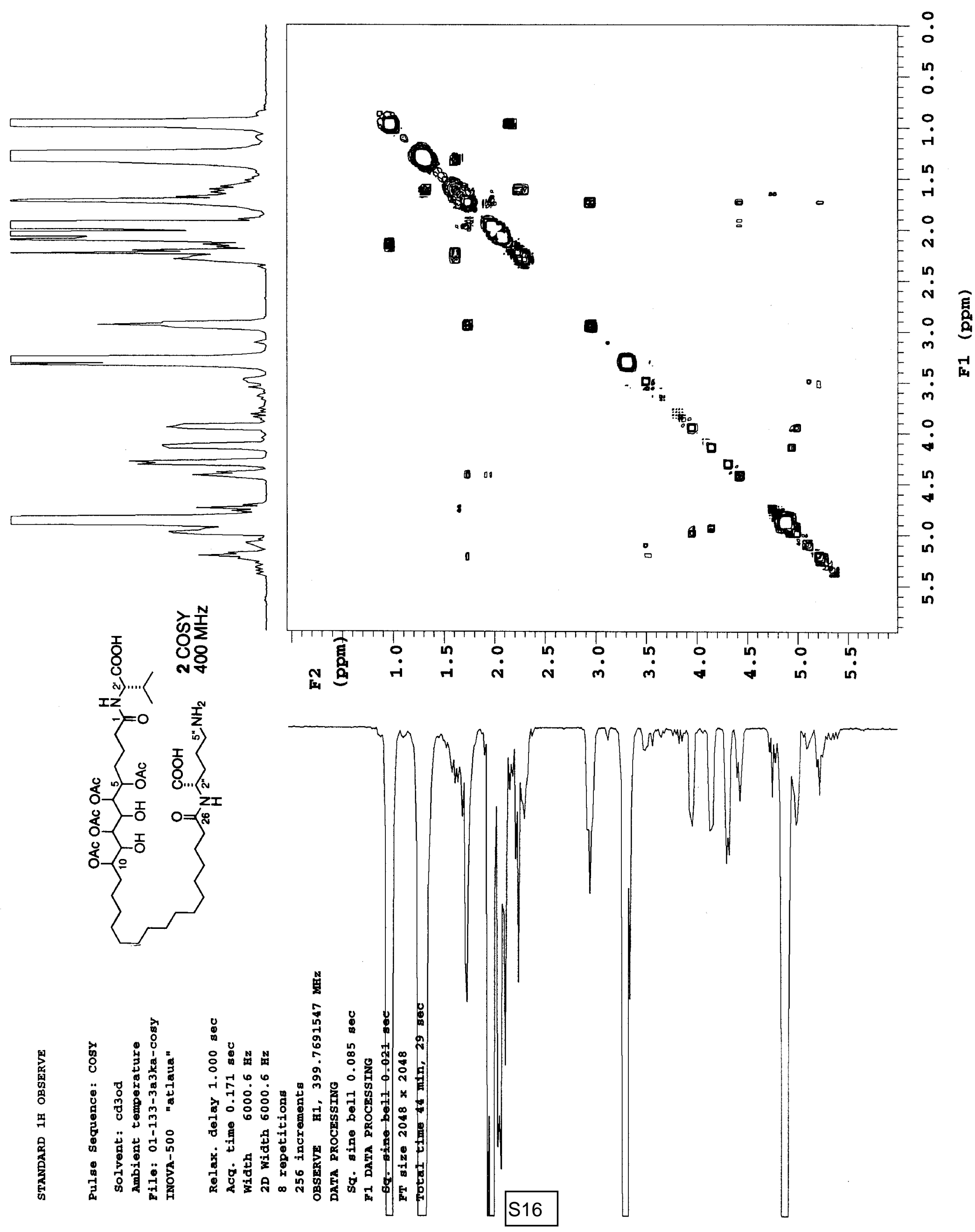




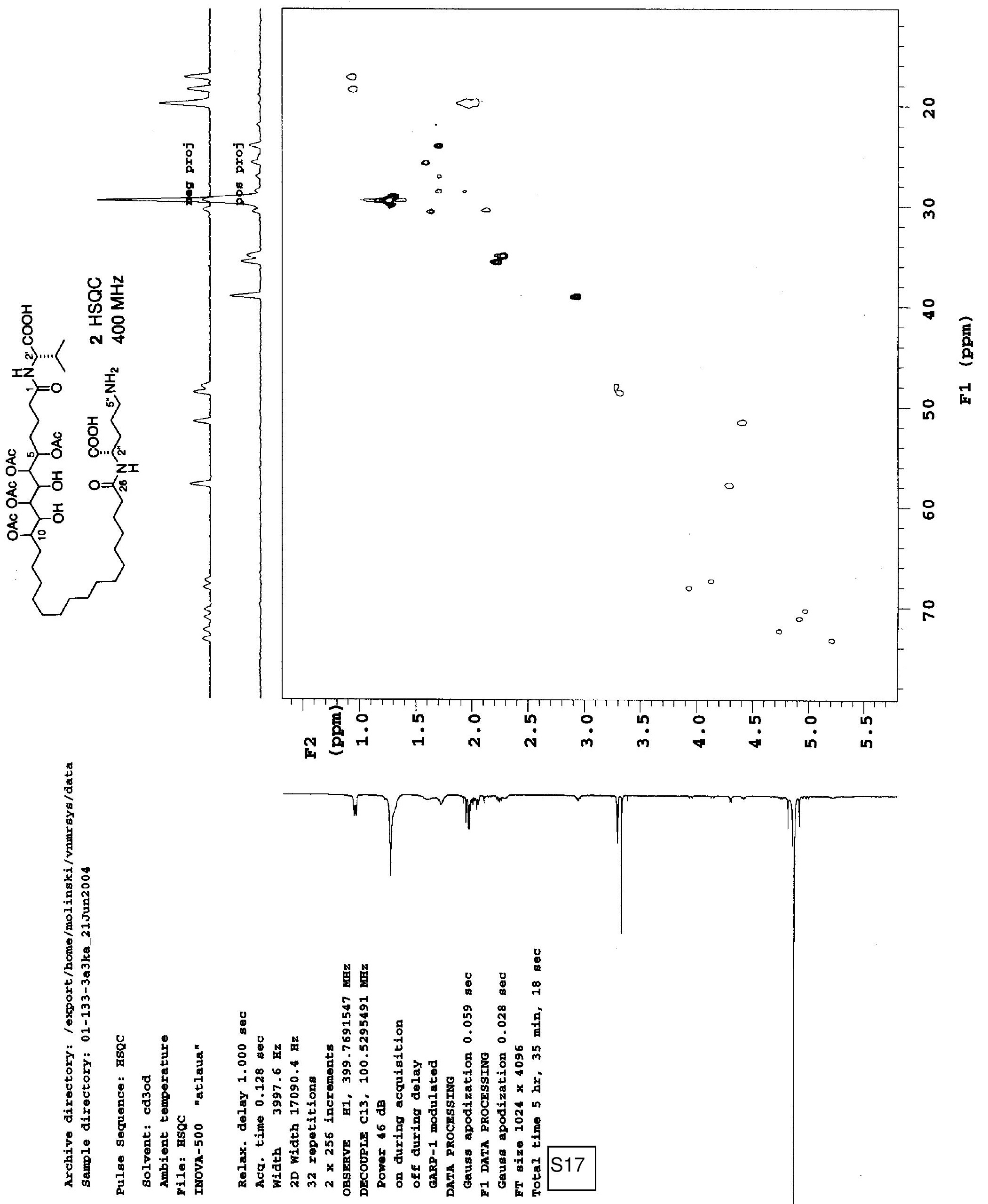




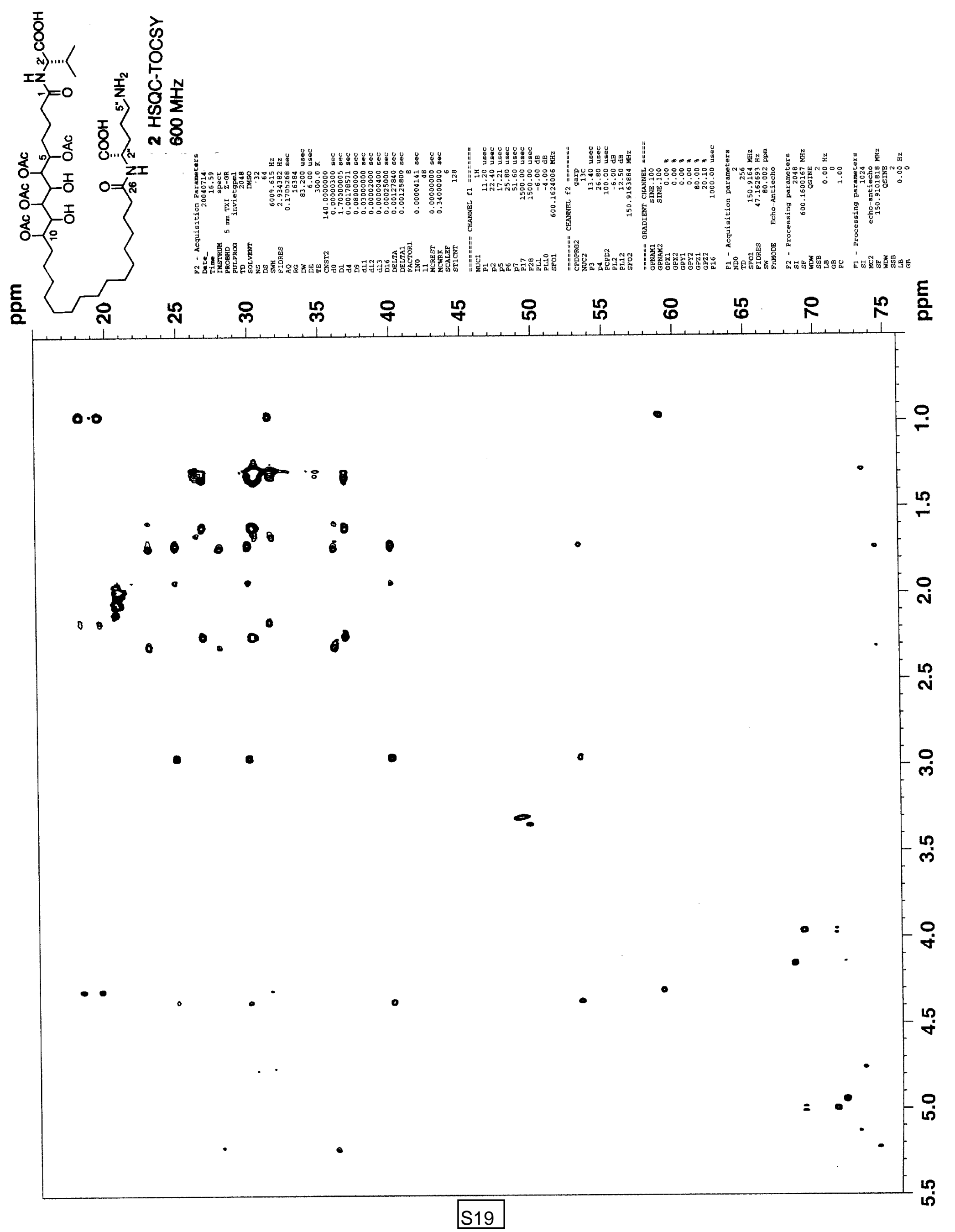

